\title{
UTILIZAÇÃO DE ACETOCHLOR E ATRAZINE APLICADOS EM MISTURA DE TANQUE COM DESSECANTES NO SISTEMA DE PLANTIO DIRETO ${ }^{1}$
}

\author{
BENEDITO N. RODRIGUES ${ }^{2}$, JOÃO DE LIMA ${ }^{3}$, INÊS F. U. YADA ${ }^{4}$ e DONIZETI A. FORNAROLLI ${ }^{5}$
}

\section{RESUMO}

A mistura em tanque de herbicidas dessecantes com residuais tem sido utilizada com frequencia pelos agricultores no sistema de plantio direto. Sabe-se, no entanto, que alguns herbicidas residuais têm problemas de retenção na palha quando utilizados isoladamente em préemergência nesse sistema. O objetivo do presente trabalho, foi estudar o comportamento dos herbicidas residuais atrazine e acetochlor em mistura com dessecantes no manejo em plantio direto. Para tal, foi instalado um experimento em blocos ao acaso com parcelas subdivididas e quatro repetições. Os tratamentos, alocados na parcela, em número de nove, foram constituidos pelas seguintes misturas de herbicidas: 1) acetochlor + glyphosate; 2) acetochlor + glyphosate $+2,4 \mathrm{D} ; 3$ ) acetochlor + paraquat; 4) acetochlor + paraquat $+2,4 \mathrm{D} ; 5)$ atrazine + glyphosate; 6) atrazine + glyphosate $+2,4 \mathrm{D} ; 7$ ) atrazine + paraquat; 8) atrazine + óleo vegetal; 9) testemunha não tratada. Os tratamentos da sub parcela, em número de dois, foram constituidos pelas modalidades de aplicação dos herbicidas na cobertura vegetal: 1) aplicação com a cobertura "em pé"; 2) aplicação com a cobertura "rolada". A cobertura vegetal de inverno era formada pela consorciação de aveia-preta + ervilhaca comum. Os resultados mostraram que o controle de Brachiaria plantaginea, Euphorbia heterophylla e Bidens pilosa foi melhor efetuado com os tratamentos onde entrou o atrazine. De maneira geral, o controle obtido na modalidade "em pé," foi melhor do que na modalidade "rolada," principalmente nos tratamentos com acetochlor. A análise cromatográfica de resíduos feita em amostras de solo retiradas do experimento antes e após uma chuva de $41 \mathrm{~mm}$ ocorrida 24 horas após a aplicação dos tratamentos, mostrou que menos de $6 \%$ do acetochlor aplicado foi detectado no solo, em ambas as modalidades de aplicação. Quanto ao atrazine, no entanto, mais de $78 \%$ do total aplicado foi detectado no solo após a chuva na modalidade "rolada", em qualquer tratamento; neste caso, os maiores valores foram $91 \%$ para atrazine+óleo vegetal e 90\% para atrazine+glyphosate+2,4D. Na modalidade "em pé", os maiores valores foram obtidos com atrazine+óleo vegetal $(77 \%)$ e com atrazine+glyphosate+2,4D (70\%).

Palavras chave: Milho, cromatografia, cobertura morta,.

\footnotetext{
${ }^{1}$ Recebido para publicação em 11/05/99 e na forma revisada em 17/09/99.

${ }^{2}$ Eng $^{\circ}$ Agr ${ }^{\circ}$ PhD, Pesquisador, Instituto Agronômico do Paraná-IAPAR. C.P. 481, CEP: 86001-970, Londrina/PR.

${ }^{3}$ Eng $^{\circ}$ Químico, MSc, Pesquisador, Instituto Agronômico do Paraná-IAPAR. C.P. 481, CEP: 86001-970, Londrina/PR.

${ }^{4}$ Eng $^{\mathrm{a}} \mathrm{Agr}^{\mathrm{a}}$, MSc, Pesquisadora, Instituto Agronômico do Paraná-IAPAR. C.P. 481, CEP: 86001 -970, Londrina/PR.

${ }^{5}$ Eng $^{\circ}$ Agr $^{\circ}$, MSc, Milenia Agro-Ciências S/A, C.P. 2025, CEP: 86031-610, Londrina/PR.
} 


\section{ABSTRACT \\ Reaction of the herbicides acetochlor and atrazine applied in tank mixtures with desiccants in no-till system}

Tank mix involving desiccants plus residual herbicides have been used by farmers in no- till system. Nevertheless, it has been commented that the residual herbicides can stay retained in the straw when used in pre-emergence in this system. The objective of this trial, was to study the reaction of the residual herbicides atrazine and acetochlor in tank mix with desiccants herbicides for corn crop in no-till system. The experiment was settled in Londrina, State of Paraná, using a randomized complete block design in a split plot arrangement. Nine treatments were located at the main plots: 1) acetochlor + glyphosate; 2) acetochlor + glyphosate $+2,4 \mathrm{D} ; 3$ ) acetochlor + paraquat; 4) acetochlor + paraquat $+2,4 \mathrm{D} ; 5$ ) atrazine + glyphosate; 6) atrazine + glyphosate $+2,4 \mathrm{D} ; 7$ ) atrazine + paraquat; 8) atrazine + vegetal oil; 9) check without herbicides. Two modalities of herbicides application of herbicides were tested in split plots: 1) application on a "non rolled" cover crops; 2) application on a "rolled"cover crops. The winter cover crops were a mixture of

\section{INTRODUÇÃO}

A utilização de herbicidas dessecantes em mistura com residuais tem sido comum entre os agricultores na operação de "manejo" no sistema de plantio direto. Essa prática pode ser vantajosa, uma vez que, numa única operação, faz-se a dessecação da cultura de inverno que vai ser utilizada como cobertura morta e também a aplicação do herbicida residual ou pré-emergente, que terá o papel de manter a cultura de verão no limpo durante parte do seu ciclo. A operação de manejo pode ser feita com a cultura de inverno "em pé" ou "rolada" (Skora Neto, 1998), sendo que ambos os métodos apresentam vantagens e desvantagens. plants formed by Avena strigosa Schreb plus Vicia sativa L. The best control of Brachiaria plantaginea, Euphorbia heterophylla and Bidens pilosa was obtained with atrazine treatments. In general terms, the treatments "non rolled" were better than the "rolled" ones for weed control. The chromatografic analysis of the soil sampled from the plots before and after $41 \mathrm{~mm}$ of a rainfall that occured $24 \mathrm{~h}$ after application of the herbicides, showed that less than $6 \%$ of the acetochlor applied was detected in soil in both modalities of application. In respect to atrazine however, more than $78 \%$ of the total applied was detected in soil after the rain in the "rolled" modality in any treatment. In this case, the highest values were obtained with atrazine + vegetal oil $(91 \%)$ and atrazine + glyphosate $+2,4 \mathrm{D}(90 \%)$. In the same manner, in "non rolled"modality, the highest values were obtained with atrazine + vegetal oil $(77 \%)$ and atrazine + glyphosate + 2,4D (70\%).

Key words: corn, chromatography, mulch.

Em trabalhos anteriores, no entanto, verificou-se que os herbicidas residuais tem comportamentos diferentes quando utilizados em pré-emergência sobre palhadas, no sistema de plantio direto. Alguns produtos como atrazine (Rodrigues \& Almeida, 1986; Fornarolli, 1997), imazaquin (Rodrigues, 1993) e sulfentrazone, possuem boas perspectivas de uso em préemergência sobre palhadas, uma vez que são facilmente lixiviados para o solo com chuvas que ocorram logo após a aplicação. Outros produtos como metribuzin (Banks \& Robinson, 1982), oryzalin (Banks \& Robinson, 1984), as acetanilidas alachlor, metolachlor e acetochlor (Banks \& Robinson, 1986), e trifluralin (Rodrigues et al., 1997), possuem problemas de retenção e/ou volatilização na palha quando 
utilizadas em pré-emergência em plantio direto. Todos esses trabalhos, no entanto, foram realizados com os herbicidas residuais aplicados isoladamente e sobre cobertura morta já formada ou "rolada", ou seja, em pré-emergência.

Dentro dessa linha de pesquisa, delineouse o presente trabalho, cujo objetivo foi estudar o comportamento dos herbicidas atrazine e acetochlor quando aplicados em mistura com dessecantes sobre cobertura vegetal no sistema de plantio direto, comparando-se as modalidades "em pé" e "rolada"

\section{MATERIAL E MÉTODOS}

O experimento foi realizado na Estação Experimental do IAPAR em Londrina, PR, no ano agrícola 1997/98.

O delineamento experimental utilizado foi o de parcelas subdivididas, sendo nove tratamentos dispostos em blocos ao acaso com quatro repetições. Os tratamentos, alocados na parcela, foram constituidos pelas seguintes misturas de herbicidas, em kg i.a/ha: 1) acetochlor + glyphosate $(2,52+0,54) ; 2)$ acetochlor + glyphosate $+2,4 \mathrm{D}$ amina $(2,52+0,54+1,0) ; 3)$ acetochlor + paraquat $(2,52+0,3) ; 4)$ acetochlor + paraquat $+2,4 \mathrm{D}(2,52+3,0+1,0) ; 5)$ atrazine + glyphosate $(2,5+0,54) ; 6)$ atrazine + glyphosate + $2,4 \mathrm{D}(2,5+0,54+1,0) ; 7)$ atrazine + paraquat $(2,5+0,3) ; 8)$ atrazine + óleo vegetal $(2,4+1,8)$; 9) testemunha não tratada. Os tratamentos alocados na subparcela, em número de dois, foram constituidos pelas modalidades de aplicação dos herbicidas na cobertura vegetal: 1) aplicação com a cobertura "em pé"; 2) aplicação com a cobertura "rolada".

Os produtos comerciais utilizados no experimento foram: Kadett CE, $840 \mathrm{~g} / \mathrm{l}$ (acetochlor); Herbitrin $500 \mathrm{BR}, 500 \mathrm{~g} / \mathrm{l}$ (atrazine); Trop, 360 g/l (glyphosate); Gramoxone, 200 g/l (paraquat); Aminol, $670 \mathrm{~g} / \mathrm{l}$ (2,4D amina); Posmil, 400+300 g/l (atrazine+óleo vegetal).

A cobertura vegetal de inverno era formada pela consorciação de aveia-preta + ervilhaca comum. A "rolagem" (passada de rolo sem facas) foi feita quinze dias antes da aplicação dos tratamentos. Estes, foram todos aplicados no mesmo dia (13 de outubro de 1997). Vinte e quatro horas após a aplicação dos tratamentos, ocorreu uma chuva natural de $41 \mathrm{~mm}$. Foram coletadas amostras de solo antes e após a chuva, com um amostrador de metal na forma de um cubo, medindo $10 \times 10 \times 10 \mathrm{~cm}$. Essas amostras foram enviadas ao Laboratório de Ecofisiologia do IAPAR, para análises cromatográficas de resíduos, visando quantificar os herbicidas residuais que chegaram ao solo antes e após a chuva.

A aplicação dos tratamentos foi feita com pulverizador de pressão constante $\left(\mathrm{CO}_{2}\right)$, munido com barra de 3 bicos espaçados 1,0m entre sí, do tipo TF 02 , vazão de 150 litros/ha. No momento da aplicação, a temperatura ambiente era de $22^{\circ} \mathrm{C}$ e a umidade relativa do ar de $86 \%$.

A cultura de verão utilizada foi o milho, semeado no sistema de plantio direto. As subparcelas tiveram as dimensões de $3 \mathrm{~m} \times 6 \mathrm{~m}$ $\left(18 \mathrm{~m}^{2}\right)$. Entre as cabeceiras e as laterais das subparcelas foram deixadas faixas não tratadas que foram utilizadas como auxiliares na avaliação visual e como fator de isolamento entre os tratamentos.

As avaliações realizadas no presente trabalho foram; 1) Fitotoxicidade dos tratamentos na cultura do milho, adotando-se a escala percentual, onde $0=$ nenhuma injúria a $100=$ morte total da cultura; 2) Porcentagem de controle das plantas daninhas, sendo $0=$ nenhum controle a $100=$ controle total; essas avaliações foram feitas aos 47 dias após a semeadura do milho e também por ocasião da colheita de grãos da cultura; 3 ) produção de grãos de milho em $\mathrm{kg} / \mathrm{ha}$; 4) Porcentagem do herbicida residual (acetochlor $\mathrm{e}$ atrazine) que chegou ao solo antes e após a chuva, determinada através de cromatografia de fase gasosa (GC).

A determinação cromatográfica de resíduos no solo e na palha foi feita conforme método descrito por Thier \& Zeumer (1987), modificado e ajustado no Laboratório de Análise 
Orgânica do IAPAR. Estes herbicídas foram extraídos do solo com acetonitrila, utilizando-se agitador orbital e purificação através de sistema de vácuo e colunas de extração de fase sólida C-18, florisil e alumina. As eluições foram realizadas com a passagem de diclorometano e concentração em rotavapor. Os resíduos foram quantificados por cromatografia de fase gasosa com injeção automática e detector termoiônico específico para nitrogênio.

Os dados obtidos, foram submetidos à análise da variância. Para a comparação de médias, foi utilizado o teste de Tukey ao nível de $5 \%$ de probabilidade.

\section{RESULTADOS E DISCUSSÃO}

Os tratamentos não provocaram fitotoxicidade na cultura do milho (dados não apresentados). Em relação ao controle da
Brachiaria plantaginea, aos 47 dias após a semeadura do milho nota-se, pela Tabela 1, que as misturas dos dessecantes com atrazine foram superiores às misturas com acetochlor no controle dessa gramínea. Verifica-se também que, de maneira geral, o controle dessa infestante foi melhor efetuado quando os tratamentos foram aplicados com a cobertura em pé do que rolada. É importante notar que a ação do atrazine não foi influenciada pelo dessecante utilizado na mistura, ou seja, não houve diferença estatística entre os tratamentos. Quanto ao acetochlor, houve diferenças significativas quando misturado com glyphosate, sendo que o controle da gramínea foi significativamente maior do que quando misturado com paraquat. Quando se introduziu 2,4D na mistura, os valores foram intermediários. Essa situação praticamente se repetiu na avaliação feita por ocasião da colheita do milho (Tabela 2).

TABELA 1. Porcentagem de controle de B. plantaginea, E. heterophylla e B. pilosa aos 47 dias após a semeadura do milho com cobertura vegetal em pé e rolada ${ }^{1}$.

\begin{tabular}{|c|c|c|c|c|}
\hline Tratamentos & Cobertura & B.plantaginea & E.heterophylla & B. pilosa \\
\hline \multirow[t]{2}{*}{ acetochlor + glyphosate } & Em pé & $86 a A B$ & $40 \mathrm{aB}$ & $75 \mathrm{aC}$ \\
\hline & Rolada & $42 \mathrm{bB}$ & $22 \mathrm{bB}$ & $65 \mathrm{bD}$ \\
\hline \multirow{2}{*}{ aceto + gly + 2,4D } & Em pé & $73 \mathrm{aBC}$ & $43 \mathrm{aB}$ & $87 \mathrm{aB}$ \\
\hline & Rolada & $20 \mathrm{bC}$ & $30 \mathrm{bB}$ & $85 \mathrm{aB}$ \\
\hline \multirow[t]{2}{*}{ acetochlor + paraquat } & Em pé & $28^{\mathrm{a}} \mathrm{D}$ & $43 \mathrm{aB}$ & $75 \mathrm{aC}$ \\
\hline & Rolada & $18 b C D$ & $23 \mathrm{bB}$ & $55 \mathrm{bE}$ \\
\hline \multirow{2}{*}{ aceto + paraquat $+2,4 \mathrm{D}$} & Em pé & $66 \mathrm{aC}$ & $47 \mathrm{aB}$ & $85 \mathrm{aB}$ \\
\hline & Rolada & $43 \mathrm{bB}$ & $20 \mathrm{bB}$ & $76 b C$ \\
\hline \multirow[t]{2}{*}{ atrazine + glyphosate } & Em pé & $96 \mathrm{aA}$ & $86 \mathrm{aA}$ & $98 \mathrm{aA}$ \\
\hline & Rolada & $88 \mathrm{aA}$ & $85 \mathrm{aA}$ & $98 \mathrm{aA}$ \\
\hline \multirow[t]{2}{*}{ atraz + gly + 2,4D } & Em pé & $97 \mathrm{aA}$ & $96 a A$ & $98 \mathrm{aA}$ \\
\hline & Rolada & $81 \mathrm{bA}$ & $85 \mathrm{bA}$ & $98 \mathrm{aA}$ \\
\hline \multirow[t]{2}{*}{ atrazine + paraquat } & Em pé & $96 a A$ & $93 \mathrm{aA}$ & $98 \mathrm{aA}$ \\
\hline & Rolada & $90 \mathrm{aA}$ & $82 \mathrm{bA}$ & $98 \mathrm{aA}$ \\
\hline \multirow[t]{2}{*}{ atrazine + óleo vegetal } & Em pé & $97^{\mathrm{a}} \mathrm{A}$ & $88 \mathrm{aA}$ & $98 \mathrm{aA}$ \\
\hline & Rolada & $91^{\mathrm{a}} \mathrm{A}$ & $87 \mathrm{aA}$ & $98 \mathrm{aA}$ \\
\hline \multirow[t]{2}{*}{ Testemunha } & Em pé & $0^{\mathrm{a}} \mathrm{E}$ & $0 \mathrm{aC}$ & $0 \mathrm{aD}$ \\
\hline & Rolada & $0^{\mathrm{a}} \mathrm{D}$ & $0 \mathrm{aC}$ & $0 \mathrm{aF}$ \\
\hline $\mathrm{CV}(\%)$ & & 11,3 & 9,0 & 3,9 \\
\hline
\end{tabular}


Utilização de acetochlor e atrazine aplicados em mistura de tanque com dessecantes no sistema de plantio direto

TABELA 2. Porcentagem de controle de B. plantaginea, E. heterophylla e B. pilosa por ocasião da colheita do milho com cobertura vegetal em pé e rolada ${ }^{1}$.

\begin{tabular}{llccc}
\hline Tratamentos & Cobertura & B.plantaginea & E. heterophylla & B. pilosa \\
\hline acetochlor + glyphosate & Em pé & $75 \mathrm{aB}$ & $43 \mathrm{aC}$ & $65 \mathrm{aC}$ \\
& Rolada & $36 \mathrm{bD}$ & $48 \mathrm{aC}$ & $53 \mathrm{bC}$ \\
aceto + gly + 2,4D & Em pé & $73 \mathrm{aB}$ & $46 \mathrm{aC}$ & $75 \mathrm{bB}$ \\
& Rolada & $36 \mathrm{bD}$ & $50 \mathrm{aC}$ & $85 \mathrm{aB}$ \\
acetochlor + paraquat & Em pé & $35 \mathrm{aD}$ & $52 \mathrm{aC}$ & $46 \mathrm{aD}$ \\
& Rolada & $7 \mathrm{bE}$ & $27 \mathrm{bD}$ & $31 \mathrm{bD}$ \\
aceto + paraquat +2,4D & Em pé & $47 \mathrm{aC}$ & $46 \mathrm{aC}$ & $45 \mathrm{aD}$ \\
& Rolada & $47 \mathrm{aC}$ & $21 \mathrm{bD}$ & $35 \mathrm{bD}$ \\
atrazine + glyphosate & Em pé & $98 \mathrm{aA}$ & $75 \mathrm{aB}$ & $96 \mathrm{aA}$ \\
& Rolada & $65 \mathrm{bB}$ & $77 \mathrm{aAB}$ & $96 \mathrm{aA}$ \\
atraz + gly + 2,4D & Em pé & $98 \mathrm{aA}$ & $90 \mathrm{aA}$ & $98 \mathrm{aA}$ \\
& Rolada & $55 \mathrm{bC}$ & $77 \mathrm{bAB}$ & $98 \mathrm{aA}$ \\
atrazine + paraquat & Em pé & $98 \mathrm{aA}$ & $96 \mathrm{aA}$ & $98 \mathrm{aA}$ \\
& Rolada & $87 \mathrm{bA}$ & $73 \mathrm{bB}$ & $98 \mathrm{aA}$ \\
atrazine + óleo vegetal & Em pé & $98 \mathrm{aA}$ & $85 \mathrm{aAB}$ & $96 \mathrm{aA}$ \\
\multirow{2}{*}{ Testemunha } & Rolada & $93 \mathrm{bA}$ & $88 \mathrm{aA}$ & $97 \mathrm{aA}$ \\
& Em pé & $0 \mathrm{aE}$ & $0 \mathrm{aD}$ & $0 \mathrm{aE}$ \\
CV $(\%)$ & Rolada & $0 \mathrm{aE}$ & $0 \mathrm{aE}$ & $0 \mathrm{aE}$ \\
\hline
\end{tabular}

${ }^{1}$ Médias seguidas de mesma letra, na coluna, não diferem estatisticamente entre si, pelo teste de Tukey a $5 \%$ de probalidade. As letras minúsculas comparam médias das modalidades de aplicação dentro de cada herbicida e as letras maiúsculas referem-se a efeitos de herbicida dentro de cada modalidade de aplicação.

Quanto a Euphorbia heterophylla e Bidens pilosa, a situação em relação ao controle foi semelhante à ocorrida com B. plantaginea. Também os tratamentos com atrazine foram superiores aos tratamentos com acetochlor no controle destas infestantes (Tabelas 1 e 2 )

A maior produção de grãos da cultura, em números absolutos, foi obtida com o tratamento atrazine + óleo vegetal aplicado com a cobertura em pé (Tabela 3). Este tratamento, no entanto, dentro da modalidade em pé, diferiu estatisticamente apenas dos tratamentos acetochlor + paraquat $+2,4 \mathrm{D}$ e do atrazine + paraquat. Estes por sua vez, não diferiram da testemunha. Dentro da modalidade rolada, não houve diferença significativa entre os tratamentos na produção de grãos.

Antes da chuva, os herbicidas residuais, como era de se esperar, pouco atingiram o solo
(Tabela 4). Destes, o atrazine teve valores superiores aos do acetochlor independentemente do dessecante utilizado na mistura. Após a chuva, o quadro não sofreu grandes alterações em relação ao acetochlor, cujas porcentagens que atingiram o solo continuaram baixas (Tabela 5). Em relação ao atrazine, no entanto, essas porcentagens foram significativamente maiores, tanto na modalidade em pé como rolada. Na modalidade em pé, o maior valor obtido no solo foi com o tratamento atrazine+óleo vegetal (77,53\% do total aplicado); este valor não diferiu estatisticamente apenas do tratamento atrazine + glyphosate + 2,4D. Na modalidade rolada, o tratamento atrazine + óleo vegetal, mais uma vez teve o maior valor $(91,75 \%)$, não diferindo também apenas de atrazine+glyphosate+2,4D, dados condizentes com os obtidos por Fornarolli (1997), utilizando atrazine. 
TABELA 3. Produção de grãos de milho em kg/ha com cobertura vegetal em pé e rolada' .

\begin{tabular}{lcc}
\hline \multicolumn{1}{c}{ Tratamentos } & Em pé & Rolada \\
\hline acetochlor+glyphosate & $2.661 \mathrm{aAB}$ & $2.598 \mathrm{aA}$ \\
aceto+glyphosate+2,4-D & $2.534 \mathrm{aAB}$ & $2.371 \mathrm{aA}$ \\
acetochlor+paraquat & $2.318 \mathrm{aAB}$ & $2.010 \mathrm{aA}$ \\
aceto+paraquat+2,4-D & $1.871 \mathrm{aB}$ & $2.033 \mathrm{aA}$ \\
atrazine+glyphosate & $2.358 \mathrm{bAB}$ & $2.772 \mathrm{aA}$ \\
atraz+glyphosate+2,4-D & $2.504 \mathrm{aAB}$ & $2.499 \mathrm{aA}$ \\
atrazine+paraquat & $2.159 \mathrm{bB}$ & $2.785 \mathrm{aA}$ \\
atrazine+óleo vegetal & $3.228 \mathrm{aA}$ & $2.595 \mathrm{bA}$ \\
testemunha & $1.806 \mathrm{bB}$ & $2.242 \mathrm{aA}$ \\
\hline
\end{tabular}

${ }^{1}$ Médias seguidas da mesma letra não diferem estatisticamente entre si, pelo teste de Tukey ao nível de 5\% de probabilidade. Letras minúsculas referem-se a efeitos de subtratamentos dentro de cada tratamento (horizontal) e, letras maiúsculas referem-se a efeitos de tratamentos dentro de cada subtratamento (vertical). C.V.=11,8\%.

TABELA 4. Porcentagem de acetochlor e atrazine que chegou ao solo antes da chuva em cobertura de aveia preta+ervilhaca comum ${ }^{1}$.

\begin{tabular}{lcc}
\hline Tratamentos & Em pé & Rolada \\
\hline acetochlor+glyphosate & $3,60 \mathrm{aE}$ & $2,60 \mathrm{aE}$ \\
aceto+glyphosate+2,4-D & $4,08 \mathrm{aE}$ & $4,45 \mathrm{aE}$ \\
acetochlor+paraquat & $3,10 \mathrm{aE}$ & $4,13 \mathrm{aE}$ \\
aceto+paraquat+2,4-D & $5,58 \mathrm{aDE}$ & $2,73^{\mathrm{a}} \mathrm{E}$ \\
atrazine+glyphosate & $11,90 \mathrm{bBC}$ & $20,78^{\mathrm{a}} \mathrm{C}$ \\
atraz+glyphosate+2,4-D & $9,60 \mathrm{bB}$ & $13,20^{\mathrm{a}} \mathrm{D}$ \\
atrazine+paraquat & $17,92 \mathrm{bA}$ & $38,23^{\mathrm{a}} \mathrm{A}$ \\
atrazine+óleo vegetal & $7,38 \mathrm{bCD}$ & $28,25^{\mathrm{a}} \mathrm{B}$ \\
\hline
\end{tabular}

${ }^{1}$ Médias seguidas da mesma letra não diferem estatisticamente entre si, pelo teste de Tukey ao nível de 5\% de probabilidade. Letras minúsculas referem-se a efeitos de subtratamentos dentro de cada tratamento (horizontal) e, letras maiúsculas referem-se a efeitos de tratamentos dentro de cada subtratamento (vertical). C.V. $=8,8 \%$.

TABELA 5. Porcentagem de acetochlor e atrazine que chegou ao solo depois da chuva em cobertura de aveia preta+ervilhaca comum ${ }^{1}$.

\begin{tabular}{lcc}
\hline \multicolumn{1}{c}{ Tratamentos } & Em pé & Rolada \\
\hline acetochlor+glyphosate & $3,03^{\mathrm{a}} \mathrm{D}$ & $2,00^{\mathrm{a}} \mathrm{C}$ \\
aceto+glyphosate+2,4-D & $2,75^{\mathrm{a}} \mathrm{D}$ & $2,63 \mathrm{aC}$ \\
acetochlor+paraquat & $3,85^{\mathrm{a}} \mathrm{D}$ & $3,33^{\mathrm{a}} \mathrm{C}$ \\
aceto+paraquat+2,4-D & $4,25^{\mathrm{a}} \mathrm{D}$ & $0,90 \mathrm{aC}$ \\
atrazine+glyphosate & $41,88 \mathrm{bC}$ & $78,43^{\mathrm{a}} \mathrm{B}$ \\
atraz+glyphosate+2,4-D & $70,43 \mathrm{bAB}$ & $90,43 \mathrm{aA}$ \\
atrazine+paraquat & $61,98 \mathrm{bB}$ & $80,05^{\mathrm{a}} \mathrm{B}$ \\
atrazine+óleo vegetal & $77,53 \mathrm{bA}$ & $91,75 \mathrm{aA}$ \\
\hline
\end{tabular}

${ }^{1}$ Médias seguidas da mesma letra não diferem estatisticamente entre si, pelo teste de Tukey ao nível de 5\% de probabilidade. Letras minúsculas referem-se a efeitos de subtratamentos dentro de cada tratamento (horizontal) e, letras maiúsculas referem-se a efeitos de tratamentos dentro de cada subtratamento (vertical). C.V. $=11,3 \%$.Obs.: Chuva natural de $41 \mathrm{~mm}$ ocorrida 24 horas após a aplicação dos tratamentos. 


\section{LITERATURA CITADA}

BANKS, P.A. \& ROBINSON, E.L. The influence of straw mulch on the soil reception and persistence of metribuzin. Weed Sci., v.30, n.2, p.164-8. 1982.

BANKS, P.A. \& ROBINSON, E.L. The fate of oryzalin applied to straw-mulched and nonmulched soils. Weed Sci., v.32, p.269-272, 1984.

BANKS, P.A. \& ROBINSON, E.L. Soil reception and activity of acetochlor, alachlor and metolachlor as affected by wheat (Triticum aestivum), straw irrigation. Weed Sci., v.34, p.607-611, 1986.

FORNAROLLI, D.A. Influência da cobertura morta no comportamento do herbicida atrazine. UEL-Londrina, PR, 1997. Dissertação de Mestrado.

RODRIGUES, B.N. Influência da cobertura morta no comportamento dos herbicidas imazaquin e clomazone. Planta Daninha, v.11, n.1 e 2, p.21-8, 1993.
RODRIGUES, B.N. \& ALMEIDA, F.S. Influência da cobertura morta no comportamento dos herbicidas atrazine e metolachlor no sistema de plantio direto. In: FUNDAÇÃO INSTITUTO AGRONÔMI-CO DO PARANÁ, Londrina, PR. Resultados de pesquisa da Área de Herbologia, safras 1984/85 e 1985/86. Londrina, 1986. (Mimeografado).

RODRIGUES, B.N.; LIMA, J; FORNAROLLI, D.A. Aplicação de trifluralin em préemergência, sobre diferentes quantidades de cobertura morta, no sistema de plantio direto.In: CONGRESSO BRASILEIRO DA CIÊNCIA DAS PLANTAS DANINHAS, 21, Caxambu, MG, 1997. Resumos... Viçosa, SBCPD, p. 365.

SKORA NETO, F. ; Manejo de plantas daninhas. In: IAPAR, ed. Plantio direto- Pequena propriedade sustentável. Londrina-PR, IAPAR, 1998. p.128-158.

THIER, H.P. \& ZEUMER, H. ed. Manual of Pesticide Residue Analysis. Weinheim: VCH, 1987. v.1, p.383-400. 